\title{
Electrical Control System of Intelligent Feed Truck Based on Programmable Logic Controller (PLC)
}

\author{
Xianying Zheng ${ }^{\mathrm{a}}$, Qingyun Meng ${ }^{\mathrm{b} \text {, * }}$ \\ Department of Mechanical Engineering, Dalian Vocational Technical College, Dalian, 116033, China \\ axy_acc@163.com, b874702490@qq.com \\ *Corresponding author
}

Keywords: PLC, Text Controller, Feed Truck

\begin{abstract}
In the breeding industry, the use of manual feeds has problems such as inefficiency and high labor costs. In view of the actual needs of the capital and factory buildings in the farm, this paper determines that the electrical system of the intelligent feed truck adopts an economical human-machine interface, which is easy to learn and convenient to maintain in the application, and is especially suitable for users with small and medium-sized farms; On this basis, the product can be modified, and the truck is suitable for feeding a variety of animals, and has certain application value.
\end{abstract}

\section{Introduction}

As we all know, due to the rapid development of the economy, the demand for various substances (clothing, medicine, etc.) is growing increasingly. The northeastern region of China is an important breeding base for various animals (minks, raccoon dogs, foxes, pigs, etc.). The animal breeding industry has developed a variety of rural operations to promote local economic development. In the past, the breeding industry used manual animal feeding, which has problems such as inefficiency and high labor costs [1]. This paper aims at the actual demand of animal feeding in scale factory of breeding industry to determine the electrical integral plan of intelligent feed truck and guide the production of equipment with the purpose of improving the working efficiency, reducing the labor cost and avoiding the waste of feed.

\section{Electrical Integral Requirements of Intelligent Feed Truck}

The feed truck is used in the farm and mainly consists of two parts of a tricycle and a feeding system. The electric tricycle is mainly used for delivery walking; the feeding system is composed of a main conveyor and an auxiliary device. After the feed is mixed and stirred uniformly, then put into the feed truck; automatic, manual and semi-automatic feeding modes are first selected and then feeding is carried out. The electrical general requirements of the intelligent feed truck are as follows:

(1) On the basis of satisfying functions, the low cost is first factor to adopt the plan.

(2) Operators have weaker operational skills and therefore higher degree of automation and reliability shall be required.

(3) The equipment must have an automatic, manual, semi-automatic mode function, which can realize a variety of optional feeding procedures, facilitate setting of the feeding amount, timely perform statistics of the amount of feeds, preset the amount of feeds, call alarm for feed shortage, hydraulic oil temperature and filter clogging, and make automatic feeding settings based on the cage width and walking speed.

\section{Control Device Plan}

The control device as the core of the electrical control system, receives all information from the 
device and enables the feed truck to move based on the preset instructions through the program execution. In accordance with the control requirements of the feed truck, the control device is optional from the following plans [2]:

(1) Single chip microcomputer (SCM) control plan: the micro-controller chip, the memory chip, the I/O interface and other chip designs constitute a digital control device. Because of the advantages of strong function, small size, high reliability, low price, etc., the single-chip microcomputer has been widely used. Its shortcoming is relatively long design time and large design workload;

(2) PLC control plan: as one of the three pillars of modern industry, PLC has been widely used in various fields. It has the characteristics of high automation, convenient maintenance, small size and relatively simple program control. With the mass application, the price of PLC is much more reasonable than in the 1980s.

(3) General purpose computer control plan: the use of a general purpose computer is more flexible, but requires dedicated software and hardware circuit conversion, which is costly and cumbersome.

According to the electrical overall demand, we can see that the use of PLC+ text display control is a more reasonable application plan.

\section{Screen and Address Settings of Text Display}

The text display, also known as the terminal display, is a human-computer interaction system simply presented in text and belongs to the human-machine interface (HMI). It has the features of simple operation, economy and practicability. The content that the PLC needs to control is written into the corresponding program, and finally displayed on the interface of the text display through the RS232 communication interface, which not only can greatly improve the convenience of operation, but also can significantly improve the work efficiency[3][4].

On the text display, the screen is edited according to the requirements of the device. The screen includes the startup screen and the human-computer interface, and the communication address of the PLC is set at the same time. According to the requirements of the intelligent feed truck, the human-computer interface of the device mainly includes initial screen, automatic, manual and semi-automatic operation, feed amount statistics, current status, alarm screen and the like. First, the initial screen is set, as shown in Figure 1. The corresponding F button is pressed to enter the settings for the split screen. The function of the F button is entered by the Page Setup - Function Key setting in the main menu, and the screen corresponding to F1-F9 is selected (Fig. 2).

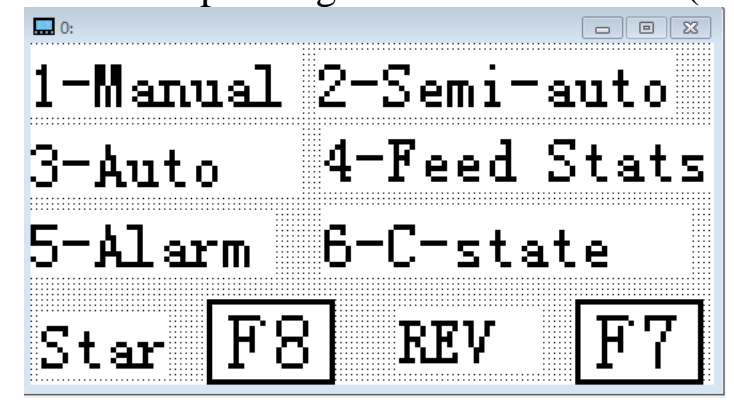

Fig.1 Initial Screen

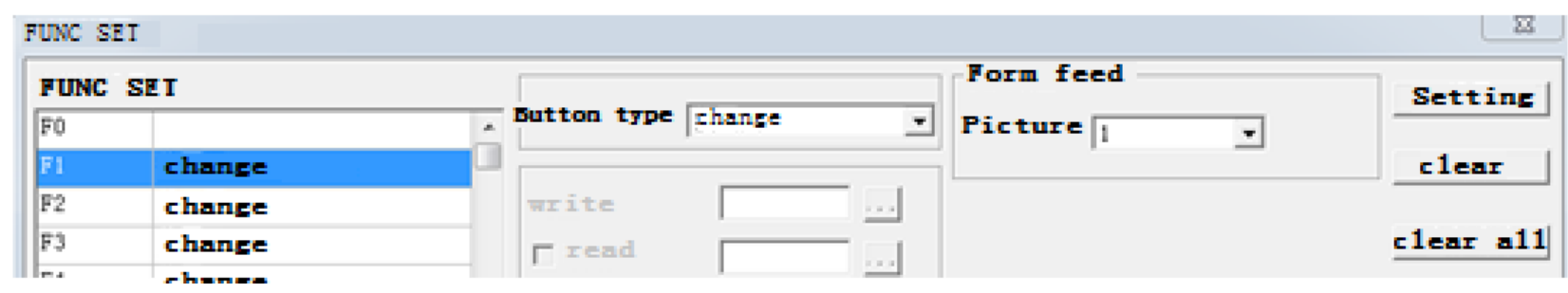

Fig.2 Functional Key Setting

After the initial screen setting is completed, the split screen is set. The static text, numerical 
display, display light, buzzer, graphics, histogram, coordinates, graphs and others can be set in each screen according to self needs. For example, the F5 button is pressed to enter the alarm screen (Fig. 3). This screen includes the oil filter clogging, text and fault display indicator for low amount of feed, high oil temperature, fault clear and forced clear. The oil filter clogging is the static text, with a signal light beside, and F1 is the functional key; the buzzer of the text display itself can be used for making sounds when the fault occurs, and then the alarm screen is entered to check what kind of fault is. In the initial screen, the F3 button is pressed to enter the automatic mode selection screen (Fig. 4). Input of the feed amount, cage spacing, and running speed is the numerical input functions. The input value setting screen is entered by double-click, as shown in Figure 5, in which the type and maximum value and minimum value of the soft component address and value associated with PLC are mainly set.

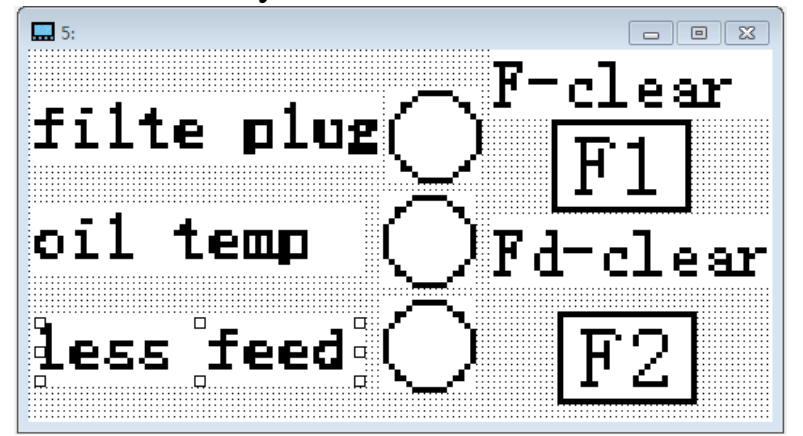

Fig.3 Alarm Screen

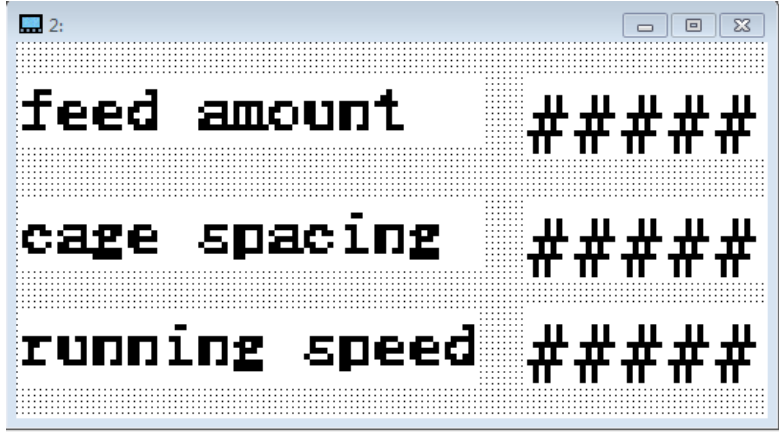

Fig.4 Automatic Setting Screen

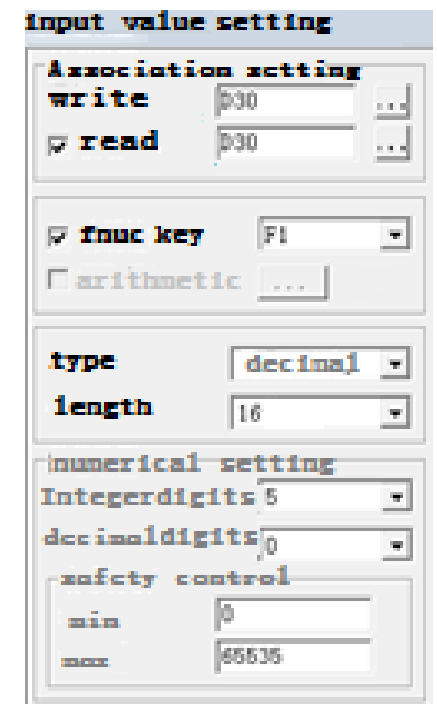

Fig.5 Input Value Setting Screen

\section{Determination of PLC Address and Program}

\subsection{Determination of I/O Address.}

The PLC is installed in the feed truck and is powered by DC. The input signals thereof are mainly switching value input signals such as foot switch, button and pressure relay, and the output is solenoid valve, and the output speed is not high. Therefore, the DC24V power supply PLC that is common in the market can be used; the I/O address layout of the PLC is shown in Figure 6[1], and the buttons such as automatic and manual are replaced by buttons on the text display[5]. 


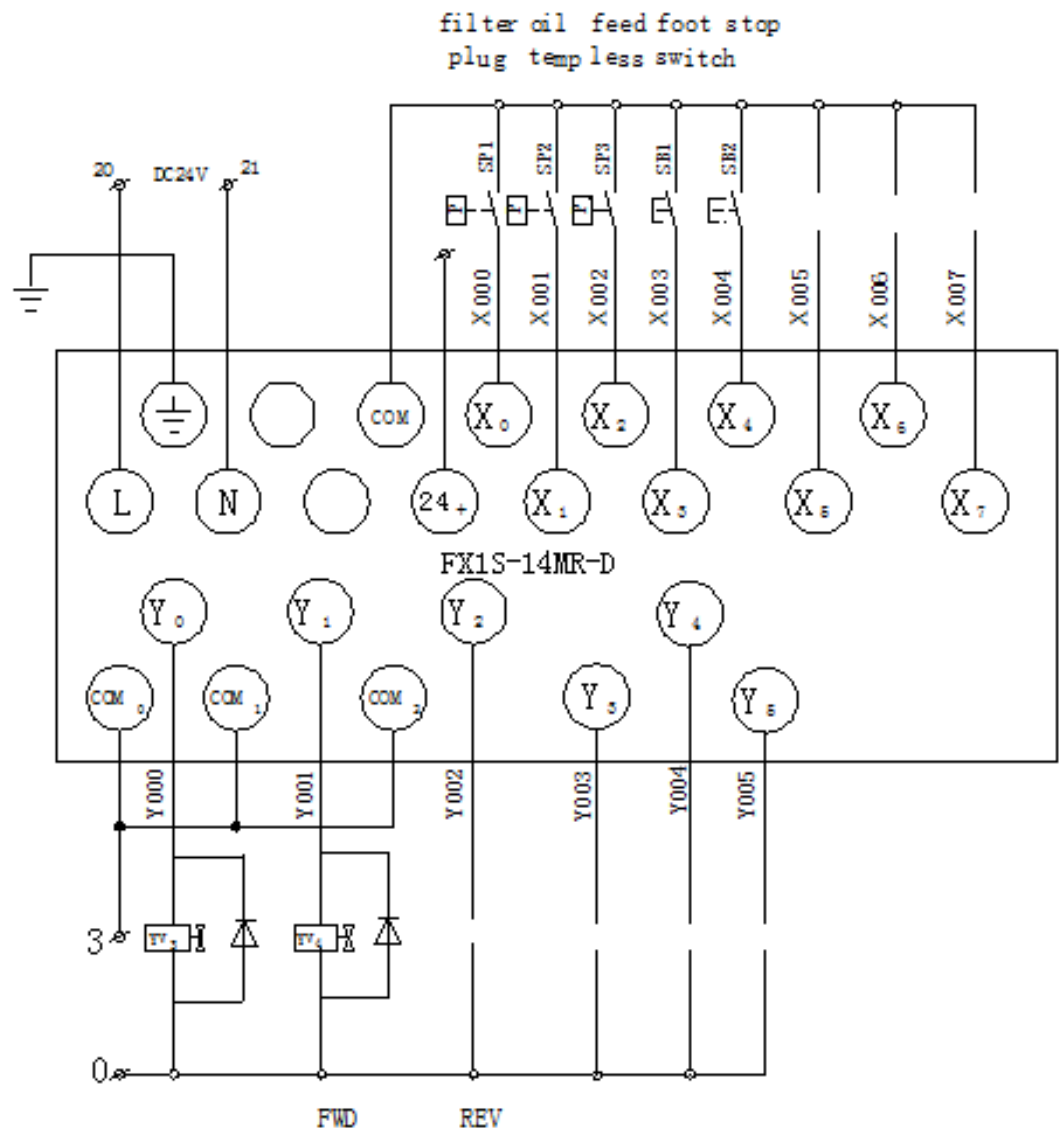

Fig.6 I/O address layout

\subsection{Program Design.}

The feed truck adopts an electric-liquid phase combination mode. The electrical system includes control of the forward and backward movement of the engine, control of the feed pump, and operation of the feed truck in manual, automatic, and semi-automatic modes. The so-called manual feeding is that the feed truck reaches the feeding location to manually control the start and stop of the feed amount. The semi-automatic feeding is that the preset feed amount is displayed on text before running of the truck, and the buttons are pressed to feed the preset feed amount after reaching the appointed location, and the truck is driven to enter the next feeding point after automatic stop. The automatic feeding is that feeding is carried out without stop of the truck, and feeding automatically starts after the start button is pressed at the first feeding point. Due to the limited space, this paper focuses on elaboration of the automatic part. In the automatic mode, the text display and the PLC communicate via the RS232 port [6], and the address of the internal register is set as follows.

Table 1 Internal register address

\begin{tabular}{|c|c|c|c|}
\hline Soft component & Comments & Soft component & Comments \\
\hline D30 & Feed amount & M50 & Fault clear \\
\hline D31 & Cage spacing & M51 & Forced clear \\
\hline D32 & running speed & M52 & Forced clear removal \\
\hline D40 & Total amount of feed & M53 & Oil filter clogging \\
\hline D41 & Current amount of feed & M54 & High oil temperature \\
\hline D0 & Flow & M55 & Less feed \\
\hline
\end{tabular}

In the automatic mode, the fixed program is set as needed. For example, the amount of feed required for the minks in different stages is different, and the program can be set according to different growth cycles or self set according to requirements. Figure 7 shows an example of a PLC based on cage spacing and running speed in an automatic situation. The reader can also modify it on 
the basis of self-actual situation.

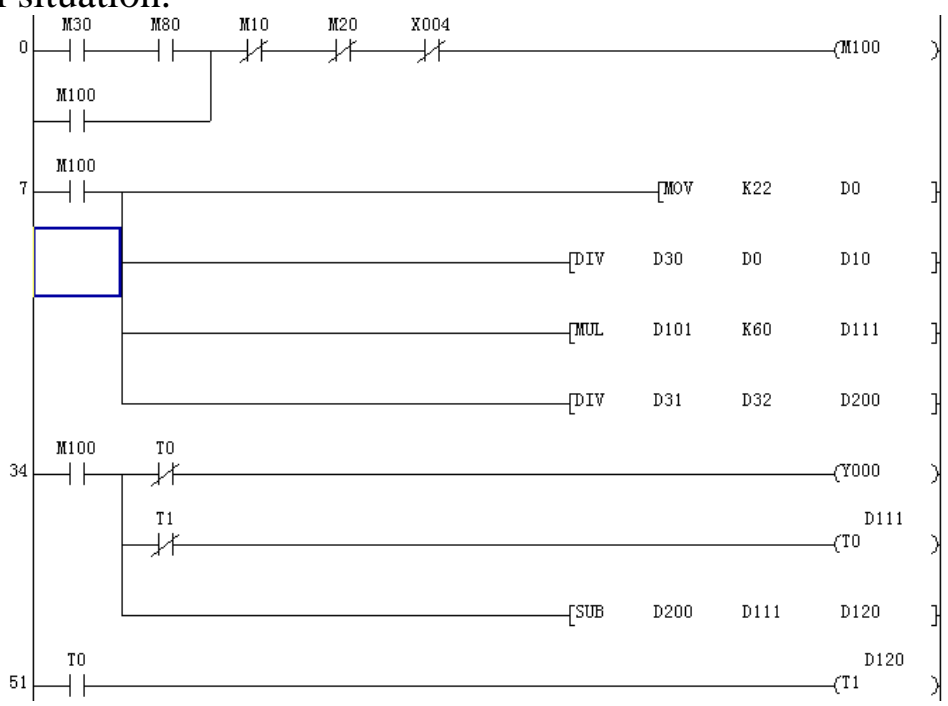

Fig.7 Ladder chart

If the feed amount is large, the length of the feed tank should be considered to avoid feeding outside the tank; the high power pump can been selected, or the hydraulic speed variator can be adopted. If the funds are sufficient, the PLC with analog auantity control can also be considered.

\section{Test Results}

This paper is related to production practice, and its purpose is to solve the problem of the labor shortage and high degree of fatigue of the operators in the present animal breeding industry. The economical electrical system is adopted to meet the needs of automatic feeding; through the adjustment of the feed amount and the statistics of the feed amount according to the specific conditions, the cost of the equipment is reduced, thereby reducing the labor cost, and the application field is wide, which is the preferred choice of the general household specialized in breeding; and the truck has certain economic benefits.

\section{Acknowledgement}

In this paper, the research was sponsored by Development of Intelligent Loading and Unloading System for Machining Center, a Key Research Subject of Dalian Vocational and Technical College (Project No.DZ2018A-01).

\section{References}

[1] Luo Wei-hong, Qin Lei, Peng Jia-chun. Current situation and Countermeasures of animal husbandry in our region. [J]. Animal husbandry and veterinary medicine. 2014:6 (31) 191-192.

[2] Chen Liang, An Yu, Xu Shan-gang. Design of a feeding system in a large-scale farm. [J]. Science and technology and innovation. 2017:14: 145-146.

[3] Ma Hong-qian, Xu Lian-ge. PLC, Frequency Converter and Touch Screen Technology and Practice [M]. Beijing: Publishing House of Electronics Industry, 2014.

[4] Zhou Zhi-min, Ji An-hua. PLC, Application and troubleshooting example of MITSUBISHI touch screen [M]. Beijing: China Electric Power Press, 2015.

[5] Wang Lie-zhun. Electrical Control and PLC Application Technology [M]. Beijing: Education Science Press, 2010.

[6] Liao Xiao-mei. Mitsubishi PLC Programming Technology and Engineering Case Selection [M]. Beijing: Mechanical Industry Press, 2012. 